\title{
Utilização da função pearson tipo V, Weibull e hiperbólica para modelagem da distribuição de diâmetros
}

\author{
Daniel Henrique Breda Binoti ${ }^{1}, 2$, Mayra Luiza Marques da Silva Binoti ${ }^{3}$, Helio Garcia Leite ${ }^{2}$ \\ ${ }^{1}$ Bolsista de Pós-doutorado do CNPq. \\ ${ }^{2}$ Universidade Federal de Viçosa, Departamento de Engenharia Florestal, Av. P.H. Rolfs, CEP 36570-000, Viçosa, MG, Brasil \\ ${ }^{3}$ Universidade Federal dos Vales do Jequitinhonha e Mucuri, Departamento de Engenharia Florestal, Rodovia MGT 367-KM 583, $n^{\circ}$ 5000, CEP 39100-000, \\ Diamantina, MG, Brasil
}

\author{
"Autor correspondente: \\ danielhbbinoti@gmail.com \\ Termos para indexação: \\ Funções de densidade \\ Probabilidade \\ Eucalipto \\ Index terms: \\ Growth and yield models \\ Probability density functions \\ Eucalyptus
}

Histórico do artigo:

Recebido em 24/08/2012

Aprovado em 01/07/2013

Publicado em 30/09/2013

doi: 10.4336/2013.pfb.33.75.426

\begin{abstract}
Resumo - Objetivou-se neste estudo avaliar a eficiência da função log-Pearson tipo V para a descrição da estrutura diamétrica de povoamentos equiâneos de eucaliptos, bem como propor um modelo de distribuição diamétrica utilizando essa função. A modelagem realizada pela função log-Pearson tipo $\mathrm{V}$ foi comparada com a modelagem realizada com a função Weibull e hiperbólica. Para isso utilizou-se dados de parcelas permanentes de eucalipto, localizadas na região centro oeste do estado de Minas Gerais. A função Pearson tipo $V$ foi testada em três diferentes configurações, com três e dois parâmetros, e tendo o parâmetro de locação substituído pelo diâmetro mínimo da parcela. A aderência das funções aos dados foi comprovada pela aplicação do teste Kolmogorov-Sminorv (K-S). Todos os ajustes apresentaram aderência aos dados pelo teste KS. As funções Weibull e hiperbólica apresentaram desempenho superior ao demonstrado pela função Pearson tipo V.
\end{abstract}

\section{Use of pearson type $V$, Weibull and hiperbolic function for modeling diameter distribution}

\begin{abstract}
The objective of this study was to evaluate the efficiency of the function log-Pearson type $\mathrm{V}$ to describe the diametric structure of even-aged stands of eucalyptus and propose a model using this function. The modeling performed by the function logPearson type $\mathrm{V}$ was compared with the modeling performed with the Weibull function and hyperbolic. It was used data from permanent plots of eucalyptus stands located in the Midwest region of Minas Gerais State, Brazil. The function of Pearson type V was tested in three different configurations, with three and two parameters, and with the parameter replaced by the minimum diameter location of the plot. Adherence functions of the data were confirmed by applying the Kolmogorov-Sminorv (KS). All adjustments to the data presented adherence by KS test. The Weibull and hyperbolic functions presented better function performance than that observed applying Pearson type V.
\end{abstract}




\section{Introdução}

A descrição das estimativas de frequência por classe diamétrica é realizada pela utilização de funções densidade de probabilidade (fdp). A escolha da fdp que melhor descreva a estrutura diamétrica do povoamento, e apresente boa flexibilidade bem como correlação significativa com características do povoamento em estudo é determinante para a qualidade das estimativas da distribuição diamétrica futura (Binoti, 2008; Campos \& Leite, 2009).

Muitas funções densidade de probabilidade têm sido utilizadas para a descrição da distribuição diamétrica de plantios florestais, dentre elas se destacam: normal de Weibull (Weibull, 1951), gama (Nelson, 1964), log-normal (Bliss \& Reinker, 1964), beta (Clutter \& Bennett, 1964) e Sb Johnson (Hafley \& Schreuder, 1977). Desde a sua introdução na área florestal, a função Weibull se tornou a mais utilizada para a modelagem da distribuição de diâmetros de povoamentos equiâneos (Campos \& Leite, 2009). Contudo, o trabalho de Binoti (2008) demonstra a existência de outras funções com superioridade sobre a função Weibull para a descrição da estrutura diamétrica de povoamentos desbastados e não desbastados.

Uma função com características interessantes e ainda não testada para a descrição da distribuição de diâmetros de povoamentos de eucalipto é a função Pearson tipo V. A função densidade de probabilidade Pearson tipo V em sua forma completa (3P) pode ser escrita da seguinte forma:

$$
f(x)=\frac{\exp \left(\frac{-\beta}{x-\alpha}\right)}{\beta \Gamma(\gamma)\left(\frac{x-\alpha}{\beta}\right)^{\gamma+1}}
$$

Em que $\gamma$ é o parâmetro de forma $(\gamma>0), \beta$ é o parâmetro de escala $(\beta>0), \alpha$ é o parâmetro de locação e $\Gamma$ é a função gama. A supressão do parâmetro de locação resulta na função Pearson tipo $\mathrm{V}$ de dois parâmetros (2P), podendo ser expressa da seguinte forma:

$$
f(x)=\frac{\exp \left(\frac{-\beta}{x}\right)}{\beta \Gamma(\gamma)\left(\frac{x}{\beta}\right)^{\gamma+1}}
$$

O parâmetro de locação também pode ser substituído pelo diâmetro mínimo da parcela (dapmin), resultando na função Pearson tipo $\mathrm{V} \operatorname{com} \alpha=$ dapmin, expressa como:

$$
f(x)=\frac{\exp \left(\frac{-\beta}{x-\text { dap } \min }\right)}{\beta \Gamma(\gamma)\left(\frac{x-\text { dap } \min }{\beta}\right)^{\gamma+1}}
$$

A função acumulativa da função Pearson tipo V, com 3 parâmetros, 2 parâmetros e com $\alpha=$ dapmin, pode ser expressa respectivamente como:

$$
\begin{gathered}
F(x)=1-\frac{\Gamma_{\left(\frac{\beta}{x-\alpha}\right)}(\gamma)}{\Gamma(\gamma)} \\
F(x)=1-\frac{\Gamma_{\left(\frac{\beta}{x}\right)}(\gamma)}{\Gamma(\gamma)} \\
F(x)=1-\frac{\Gamma_{\left(\frac{\beta}{x-d a p \min }\right)}(\gamma)}{\Gamma(\gamma)}
\end{gathered}
$$

Objetivou-se neste estudo avaliar a eficiência da função Pearson tipo $\mathrm{V}$, nas três configurações apresentadas (Johnson et al., 1994), para a descrição da estrutura diamétrica de povoamentos eqüiâneos, bem como propor um modelo de distribuição diamétrica utilizando tais funções.

\section{Material e métodos}

Os dados utilizados nesse estudo foram obtidos de 400 parcelas retangulares permanentes de $340 \mathrm{~m}^{2}$, instaladas em povoamentos de híbridos de Eucalyptus grandis $\mathrm{x}$ Eucalyptus urophylla, na região Centro Oeste do Estado de Minas Gerais. As medições de diâmetros foram efetuadas à 1,3 de altura do solo (DAP) nas árvores, com diâmetro acima de $5 \mathrm{~cm}$, nas idades médias de 28 , $40,52,64,76$ e 84 meses.

Os dados de DAP observados em cada parcela foram agrupados em classes de diâmetro com amplitude de $1,0 \mathrm{~cm}$. Ajustou-se a função Pearson tipo V nas formas: Pearson tipo V 3P, Pearson tipo V 2P e Pearson tipo $\mathrm{V} \alpha=$ dapmin .

Ajustou-se, também, um modelo de distribuição de diâmetros utilizando a função Weibull (7) e hiperbólica (8), descritas como:

$$
f(x)=\frac{\gamma}{\beta}\left(\frac{x}{\beta}\right)^{\gamma-1} e^{\left(-\left(\frac{x}{\beta}\right)^{\gamma}\right)}
$$




$$
f(X)=\frac{\gamma}{\beta}\left(\frac{x}{\beta}\right)^{(\gamma-1)}\left(1-\tanh \left(\left(\frac{x}{\beta}\right)^{\gamma}\right)^{2}\right)
$$

Em que $\beta$ é o parâmetro de escala $(\beta>0)$ e $\gamma$ é o parâmetro de forma $(\gamma>0)$.

Os ajustes foram feitos em planilha eletrônica, pelo método da máxima verossimilhança, com auxílio de técnicas de Visual Basic for Applications. Modelos de distribuição diamétrica utilizando a função Weibull e hiperbólica foram construídos para fins comparativos.

Para avaliar a aderência da função aos dados utilizouse o teste Kolmogorov-Smirnov (K-S) com 99\% de probabilidade (Sokal \& Rohlf, 1981). Realizou-se a análise gráfica entre valores estimados e observados para todos os ajustes obtidos com a função Pearson tipo $\mathrm{V}$, Weibull e hiperbólica. Avaliou-se também os ajustes pela soma de quadrados dos desvios entre os valores observados e estimados por classe de diâmetro (SQD):

$$
S Q D=\sum_{1}^{n}(Y-\hat{Y})^{2}
$$

A construção do modelo de distribuição diamétrica baseou-se na alternativa de correlação dos parâmetros da fdp com características do povoamento. Conforme Nogueira et al. (2005), utilizou-se como variáveis dependentes os parâmetros da função log-logística em uma idade futura, e como variáveis independentes os parâmetros da função e características do povoamento em uma idade atual.Construiu-se um modelo para cada configuração testada da função. O coeficiente de correlação entre os valores observados e estimados e a análise gráfica dos resíduos foram utilizados para avaliar a exatidão das estimativas do sistema de equações.

\section{Resultados}

Ajustou-se as funções Pearson tipo V, Weibull e hiperbólica para todas as parcelas e em cada situação e em cada configuração, sendo cada estimação comparada com a distribuição observada. Para cada função obtevese 400 ajustes, totalizando 2.000 ajustes.

A aderência das funções aos dados foi comprovada pelo teste Kolmogorov-Smirnov $(\alpha>0,01)$. Os valores médios da estatística do teste e a soma de quadrados dos desvios entre valores observados e estimados por classe de diâmetro é apresentado na Tabela 1. Para ambos os testes, a função hiperbólica apresentou resultados superiores, seguida pela função Weibull. A função Pearson tipo V em todas as configurações testadas apresentou valores inferiores às demais funções testadas.

Tabela 1. Valores médios da estatística do teste KolmogorovSmirnorv (dn) e soma de quadrados dos desvios (SQD) para as funções Weibull, hiperbólica, Pearson tipo V (2P), Pearson tipo V (3P), Pearson tipo V ( $\alpha=$ dapmin $)$.

\begin{tabular}{lcc}
\hline Função & dn & SQD \\
\hline Hiperbólica & 0,0514 & 139,457 \\
Weibull & 0,0526 & 140,539 \\
Pearson tipo 5 2P & 0,1139 & 523,959 \\
Pearson tipo 5 3P & 0,0780 & 293,422 \\
Pearson tipo 5 $\gamma=$ dapmin & 0,1843 & 1107,268 \\
\hline
\end{tabular}

Os modelos de distribuição diamétrica ajustados com seus respectivos coeficientes de correlação são apresentados na Tabela 2. Para as características do povoamento, ajustaram-se os modelos para diâmetro máximo e mínimo, e não houve a necessidade de estimação do número de árvores, pois não houve mortalidade significativa.

A dispersão gráfica dos resíduos percentuais e os histogramas da dispersão dos erros para cada equação que compõe o sistema são apresentados na Tabela 3.

\section{Discussão}

A capacidade da função Pearson tipo V na descrição da distribuição de diâmetros de povoamentos equiâneos de eucalipto, foi avaliada com a função em três configurações: Pearson tipo V com 3 parâmetros, Pearson tipo V com 2 parâmetros, sendo o parâmetro de locação suprimido, e com o parâmetro de locação substituído pelo diâmetro mínimo da parcela. A capacidade de projeção dos parâmetros foi avaliada, a fim de qualificar a função para a utilização em modelos de distribuição diamétrica. Modelos de distribuição diamétrica utilizando a função Weibull e hiperbólica foram construídos para fins comparativos.

A função Pearson tipo $\mathrm{V}$ apresentou, em todas as configurações testadas, valores inferiores de K-S e SQD que as funções Weibull e hiperbólica, conforme observado na Tabela 1. Em relação à avaliação da projeção da distribuição diamétrica, observa-se que em todas as configurações a função Weibull e hiperbólica apresentaram melhores dispersão gráficas dos resíduos, sendo estes não tendenciosos e bem distribuídos, conforme observado na Tabela 3.

A função Weibull apresenta considerável tradição na área florestal, sendo amplamente utilizada em modelos de distribuição de diâmetros. Essa função possui como principal característica a sua flexibilidade e a 
correlação significativa, normalmente encontrada entre seus parâmetros e características dos povoamentos (Campos \& Leite, 2009). A função hiperbólica é relativamente nova, e foi introduzida na área florestal por Leite et al. (2010). A função apresenta flexibilidade superior a demonstrada pela função Weibull, sendo que seus parâmetros apresentam boa correlação com características do povoamento.

Tabela 2. Modelos de distribuição diamétrica ajustados com seus respectivos coeficientes de correlação

\begin{tabular}{|c|c|}
\hline Pearson tipo V3P & Coeficiente de correlação $\left(R_{\hat{y y}}\right)$ \\
\hline$\gamma_{2}=\gamma_{1} e^{\left(-0,0003\left(I_{2}^{1,6106}-I_{1}^{1,6106}\right)\right)}$ & 0,8278 \\
\hline$\beta_{2}=\beta_{1} e^{\left(-0,00002\left(I_{2}^{2,0551}-I_{1}^{2,0551}\right)\right)}$ & 0,7666 \\
\hline$\alpha_{2}=\alpha_{1} e^{\left(-19,8944\left(I_{2}^{-1,0257}-I_{1}^{-1,0257}\right)\right)}$ & 0,8606 \\
\hline Pearson tipo V 2P & Coeficiente de correlação $\left(R_{\hat{y y}}\right)$ \\
\hline$\gamma_{2}=\gamma_{1} e^{\left(-0,1460\left(I_{2}^{0,5256}-I_{1}^{0,5256}\right)\right)}$ & 0,9703 \\
\hline$\beta_{2}=\beta_{1} e^{\left(-0,0002\left(I_{2}^{1,6454}-I_{1}^{1,6454}\right)\right)}$ & 0,9683 \\
\hline Pearson tipo $V \boldsymbol{\alpha}=$ dapmin & Coeficiente de correlação $\left(R_{\hat{y y y}}\right)$ \\
\hline$\gamma_{2}=\gamma_{1} e^{\left(-0,0116\left(I_{2}^{0,9510}-I_{1}^{0,9510}\right)\right)}$ & 0,7114 \\
\hline$\beta_{2}=\beta_{1} e^{\left(-2911,053\left(I_{2}^{-2,9451}-I_{1}^{-2,9451}\right)\right)}$ & 0,6252 \\
\hline Weibull & Coeficiente de correlação $\left(R_{\hat{y y y}}\right)$ \\
\hline $\ln \gamma_{2}=\ln \gamma_{1} e^{\left(-(0,7429)\left(I_{2}^{0,1318}-I_{1}^{0,1318}\right)\right)}$ & 0,9818 \\
\hline$\beta_{2}=\beta_{1}\left(\frac{I_{1}}{I_{2}}\right)+20,7092\left(1-\frac{I_{1}}{I_{2}}\right)$ & 0,9551 \\
\hline Hiperbólica & Coeficiente de correlação $\left(R_{\hat{y} \mathrm{y}}\right)$ \\
\hline $\ln \gamma_{2}=\ln \gamma_{1} e^{\left(-0,0001\left(I_{2}^{1,6906}-I_{1}^{1,6906}\right)\right)}$ & 0,9824 \\
\hline$\beta_{2}=\beta_{1}\left(\frac{I_{1}}{I_{2}}\right)+21,4228\left(1-\frac{I_{1}}{I_{2}}\right)$ & 0,9570 \\
\hline Características do povoamento & Coeficiente de correlação $\left(R_{\hat{y} y}\right)$ \\
\hline$d \max _{2}=d \max _{1} e^{\left(-4,7152\left(I_{2}^{-0,2158}-I_{1}^{-0,2158}\right)\right)}$ & 0,9743 \\
\hline $\operatorname{dmin}_{2}=\operatorname{dmin}_{1} \mathrm{e}^{\left(-3,3042\left(I_{2}^{-0,6399}-I_{1}^{-0,6399}\right)\right)}$ & 0,9517 \\
\hline
\end{tabular}

Em que, $I_{1}$ e $I_{2}$ são as idades atual e futura, respectivamente, em meses; $\gamma_{1}$ e $\gamma_{2}$ são os parâmetros de forma das funções testadas nas idades atual e futura; $\beta_{1}$ e $\beta_{2}$ são os parâmetros de escala das funções testadasnas idades atual e futura; $\alpha_{1}$ e $\alpha_{2}$ são os parâmetros de locação da função log-logística nas idades atual e futura; $\operatorname{dmax}_{1}$ e dmax 2 são os diâmetros máximos nas idades atual e futura, em cm, dmin ${ }_{1}$ e dmin 2 são os diâmetros mínimos nas idades atual e futura, em $\mathrm{cm}$, e ln é o logaritmo neperiano. 
A modelagem de distribuições de diâmetros em plantios de clones de eucaliptos tem sido feita com base em equações de predição e ou projeção de parâmetros de uma distribuição estatística (Nogueira et al., 2005; Campos \& Leite, 2009; Binoti et al., 2011b). A função Weibull de dois parâmetros tem sido utilizada na maioria dos estudos devido a suas características de flexibilidade e da facilidade de correlação de seus parâmetros com características dos povoamentos (Binoti et al., 2011a; 2011b).
As funções foram ajustadas utilizando o sistema FitFD (http://neuroforest.ucoz.com/), pelo o método da máxima verossimilhança. Outras formas de ajuste, como o método dos percentis (Guimarães, 1994), momentos (Frazie, 1981) e momentos-1 devem ser avaliadas, a fim de determinar a melhor forma de ajuste das funções hiperbólica e Pearson tipo V.

Tabela 3. Dispersão e histograma de resíduos em função da idade do povoamento para as equações que compõe o modelo de distribuição de diâmetros.

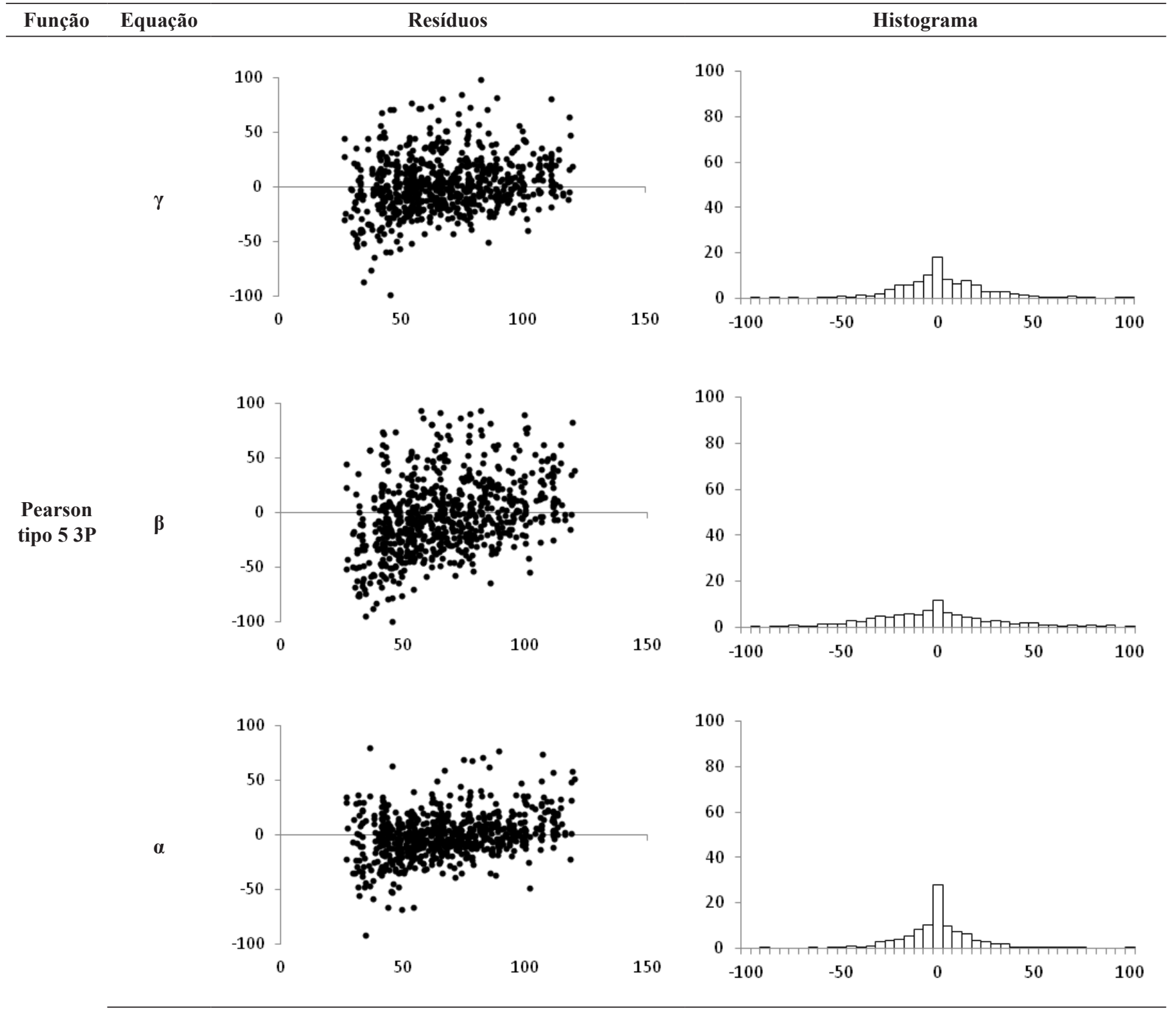


Tabela 3. Continuação.

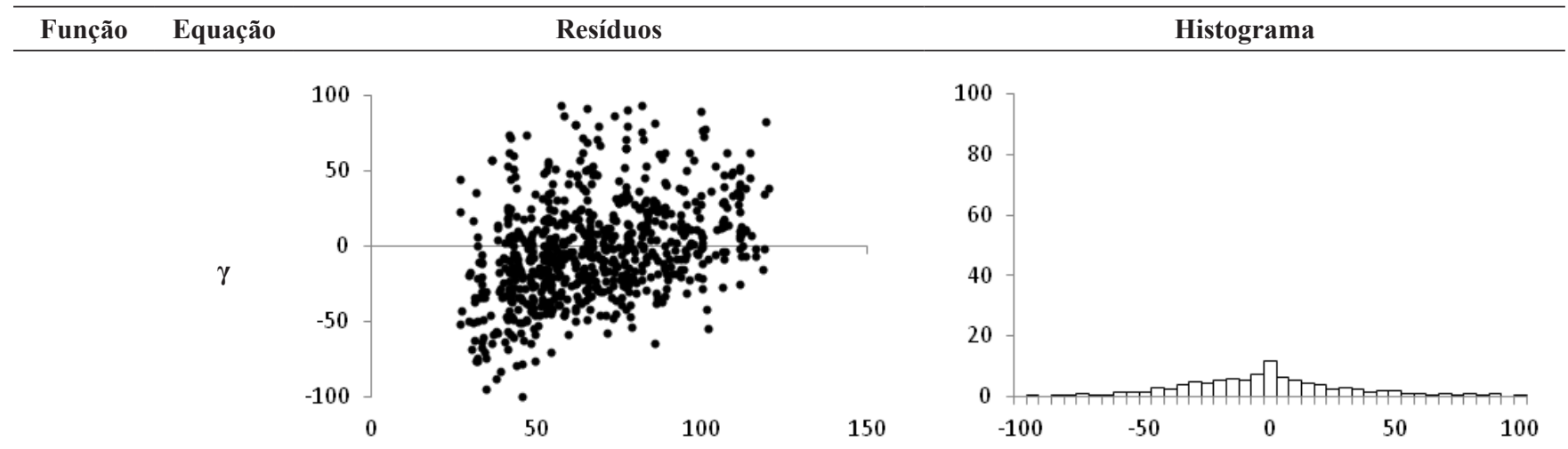

Pearson

tipo $52 \mathrm{P}$

$\alpha$
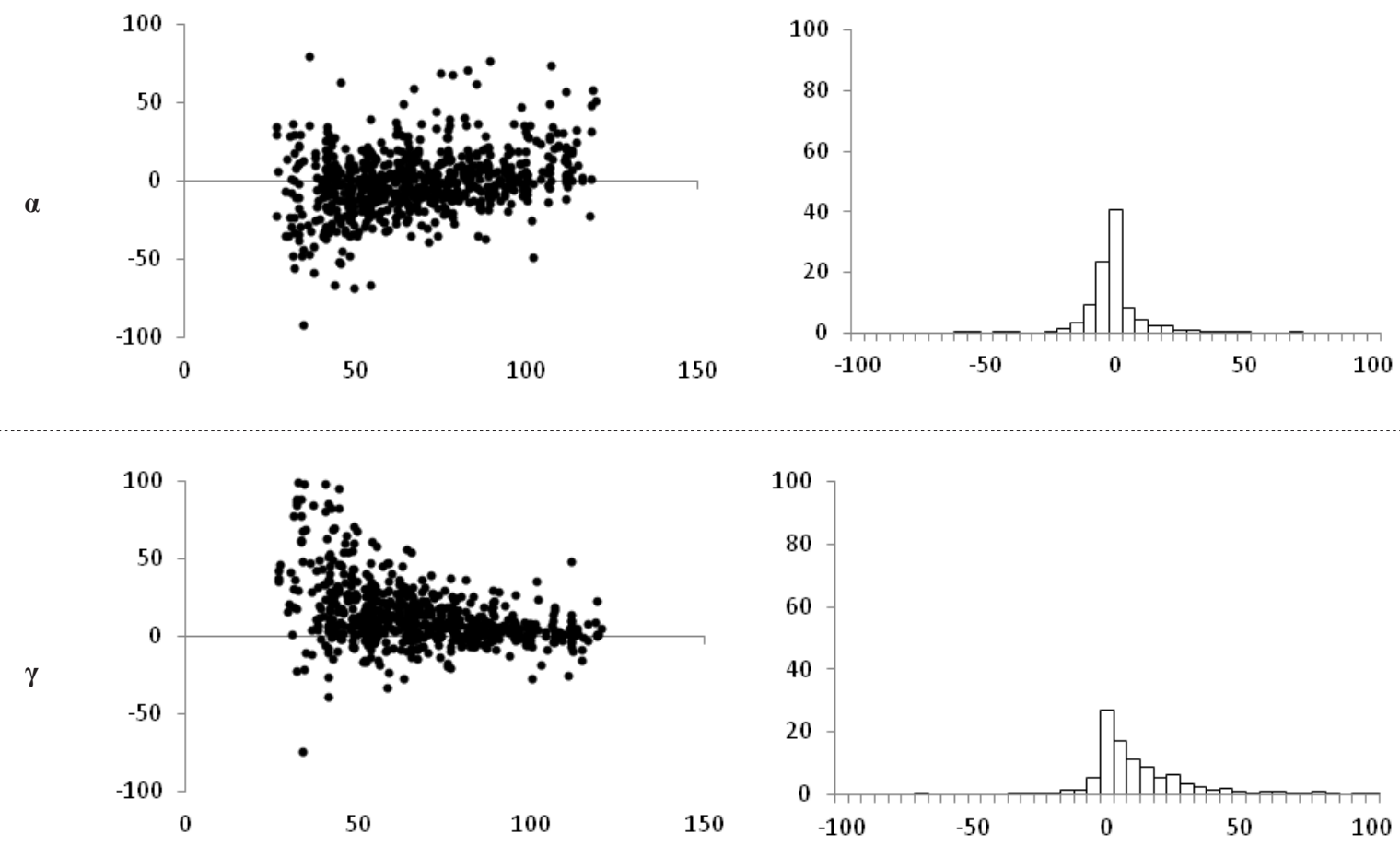

Pearson

tipo 5

$\gamma=$ dapmin
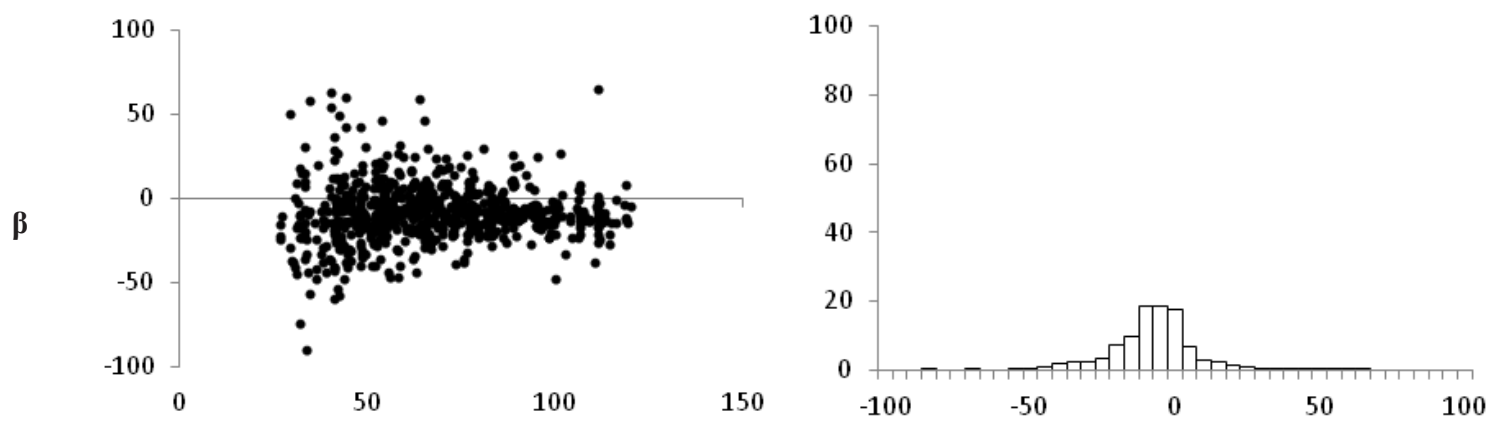
Tabela 3. Continuação.

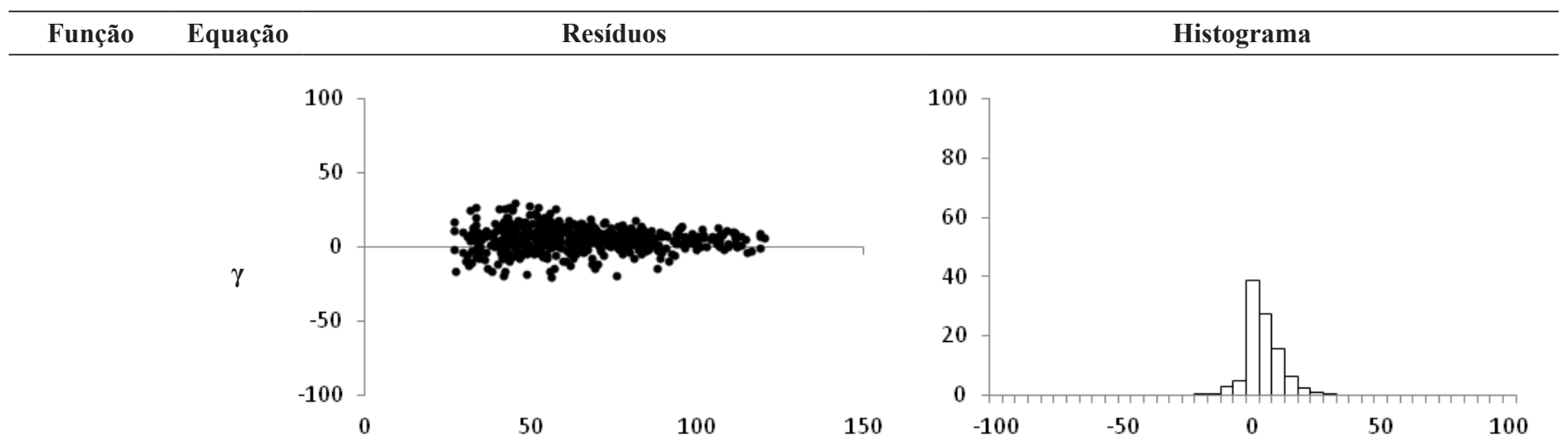

Weibull
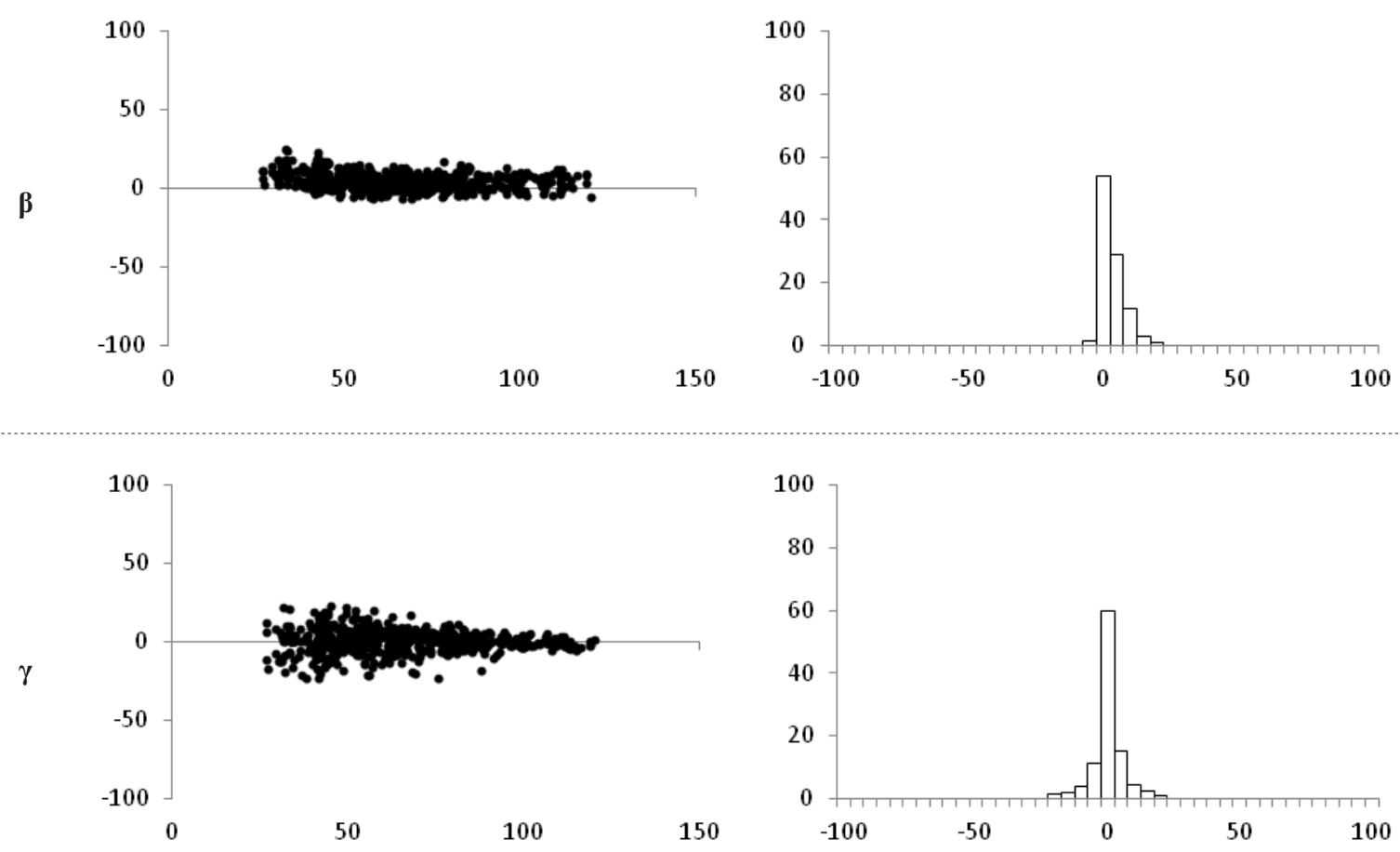

Hiperbólica
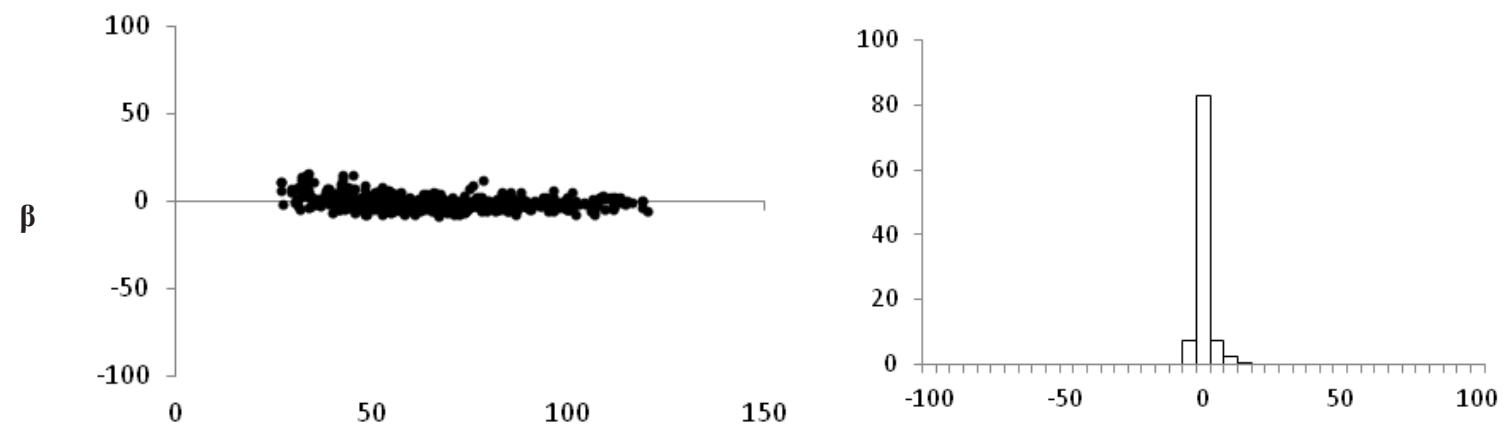
Tabela 3. Continuação.

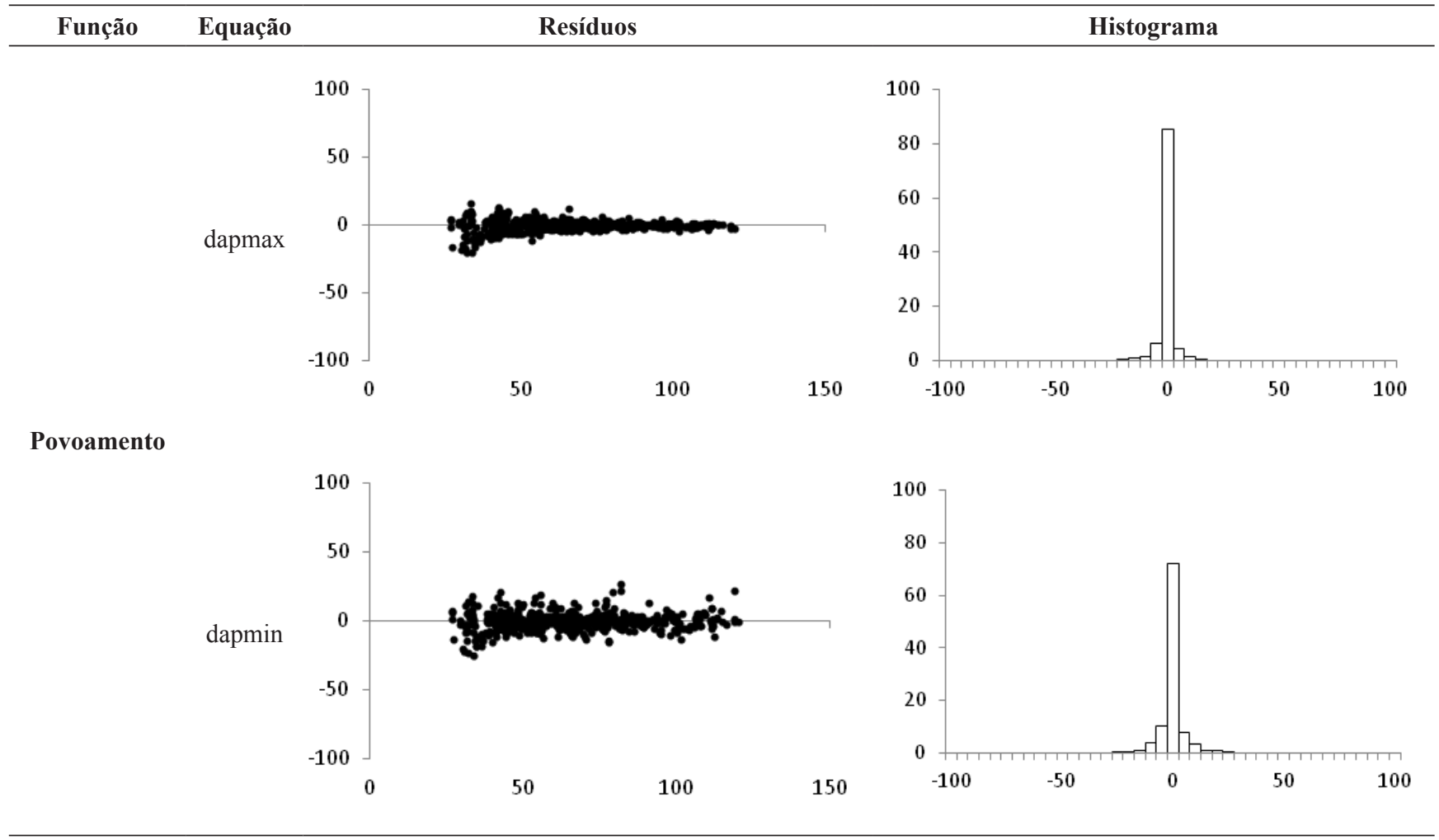

\section{Conclusões}

A função Pearson tipo V apresentou desempenho inferior à função Weibull e hiperbólica, quanto a descrição e projeção da distribuição diamétrica de povoamentos equiâneos de eucalipto.

\section{Referências}

BINOTI, D. H. B. Funções densidade de probabilidade para a descrição da distribuição diamétrica de povoamentos desbastados de Tectona grandis. 2008. 42 f. Monografia (Graduação em Engenharia Florestal) - Universidade Federal de Viçosa, Viçosa, MG.

BINOTI, D. H. B.; BINOTI, M. L. M. S.; LEITE, H. G.; GARCIA, S. L. R.; CRUZ, J. P. Modelos de distribuição diamétrica para povoamentos desbastados de eucalipto utilizando a função hiperbólica. Revista Árvore, Viçosa, MG, v. 35, p. 1069-1076, 2011 b.

BINOTI, D. H. B.; LEITE, H. G.; GUIMARÃES, D. P.; SILVA, M. L. M.; GARCIA, S. L. R.; FARDIN, L. P. Eficiência das funções weibull e hiperbólica para descrição de distribuições diamétricas de povoamentos de Tectona grandis. Revista Árvore, Viçosa, MG, v. 35 , p. 299-306, 2011a.
CAMPOS, J. C. C.; LEITE, H. G. Mensuração florestal: perguntas e respostas. 3 ed. rev. e ampl. Viçosa, MG: Ed. UF, 2009. 548 p.

FRAZIE, J. R. Compatible whole-stand and diameter distribuition models for loblolly pine plantations. 1981. $125 \mathrm{f}$. Thesis (Ph. D.) Virginia Polytechnic Institute and State University, Blackburg, VA.

GUIMARÃES, D. P. Desenvolvimento de um modelo de distribuição diamétrica de passo invariante para prognose e projeção da estrutura de povoamentos de eucalipto. 1994. 160 f. Tese (Doutorado em Ciência Florestal) - Universidade Federal de Viçosa, MG.

JOHNSON, N. L.; KOTZ, S.; BALAKRISHNAN, N. Continuous univariate distributions. 2nd ed. New York: John Wiley \& Sons, 1994. 769 p. v. 1.

LEITE, H. G.; BINOTI, D. H. B.; GUIMARÃES, D. P.; SILVA, M. L. M.; GARCIA, S. L. R. Avaliação do ajuste das funções Weibull e hiperbólica a dados de povoamentos de eucalipto submetidos a desbaste. Revista Árvore, Viçosa, MG, v. 34, n. 2, p. 305- 311, 2010.

NOGUEIRA, G. S.; LEITE, H. G.; CAMPOS, J. C. C.; CARVALHO, A. F.; SOUZA, A. L. de. Modelo de distribuição diamétrica para povoamentos de Eucalyptus sp. submetidos a desbaste. Revista Árvore, MG, v. 29, n. 4, p. 579-589, 2005.

SOKAL, R. R.; ROHLF, F. J. Biometry. San Francisco: Freeman. 1981. 